\title{
Counterintuitive MCNPX Results for Scintillator Surface Roughness Effect
}

\section{Ding Yuan a and Paul Guss ${ }^{b}$}

aNational Security Technologies, LLC, Los Alamos Operations, P.O. Box 809, M/S LAO/C320, Los Alamos, NM 87544

${ }^{b}$ National Security Technologies, LLC, Remote Sensing Laboratory, P.O. Box 98521, M/S RSL-48, Las Vegas, NV 89193

\section{SPIE Optics + Photonics}

Penetrating Radiation System and Applications XIII (OP328)

\section{August 12-16, 2012 San Diego, CA USA}

This work was done by National Security Technologies, LLC, under Contract No. DE-AC52-06NA25946 with the U.S. Department of Energy and supported by the Site-Directed Research and Development Program. 


\section{Why a Surface Roughness Study?}

- Scientific Curiosity

- Most solid radiation detection materials, in particular solid scintillation materials, produced by current industrial processes have smooth surfaces. No one has looked at alternative surface types.

- Common Sense Analogies

- Sanded glass is less reflective than smooth glass. Could this mean more energy is deposited on the sanded glass (or is there simply more scattering)?

- An open window in a distant wall appears dark. Apparently this traps more photons than a flat wall. Can we create some tiny windows on a detector's surface to trap more incoming particles?

- Blackbodies (practically a deep cavity) absorb incoming photons and converts their energy into thermal energy (increased temperature + thermal emission output). Could some kind of surface cavity structure help to improve the scintillation output? 


\section{Why Choose Inorganic Scintillation Detectors}

- Scientific / Technical / Industrial Considerations

- Scintillators convert the kinetic energy of charged particles into detectable light-more energy deposited implies more scintillation light output. (G. Knoll, 2000)

- Scintillation detectors, in particular Nal detectors, are the most widely-used radiation detectors. Therefore, a surface roughness study, if successful, would have an immediate industrial impact.

- $\mathrm{LaBr}_{3}$ somehow represents a current trend in inorganic scintillator studies. If a roughness study proves useful for $\mathrm{LaBr}_{3}$, then its application base is broadened. 


\section{Primarily Considering $\varphi 1$ 1"x1" Scintillation Crystals}

\section{Ф1" X1" Scintillation Crystal Geometrical Properties}

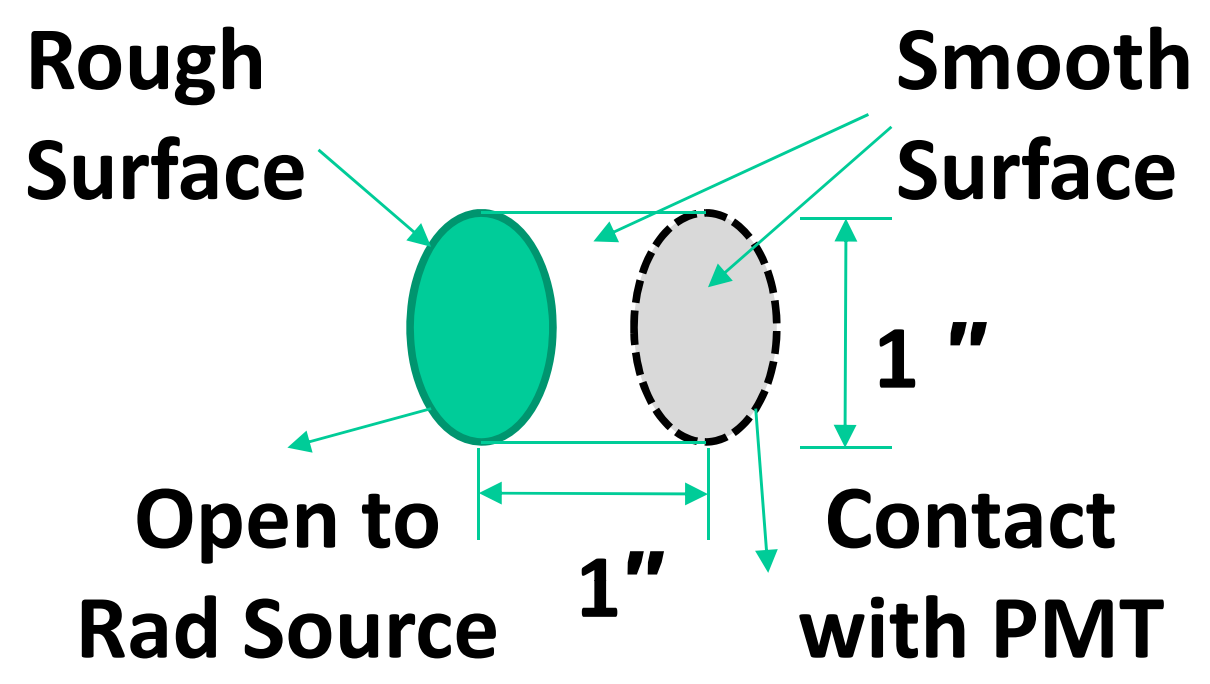




\section{MCNPX Settings Aluminum Casing}

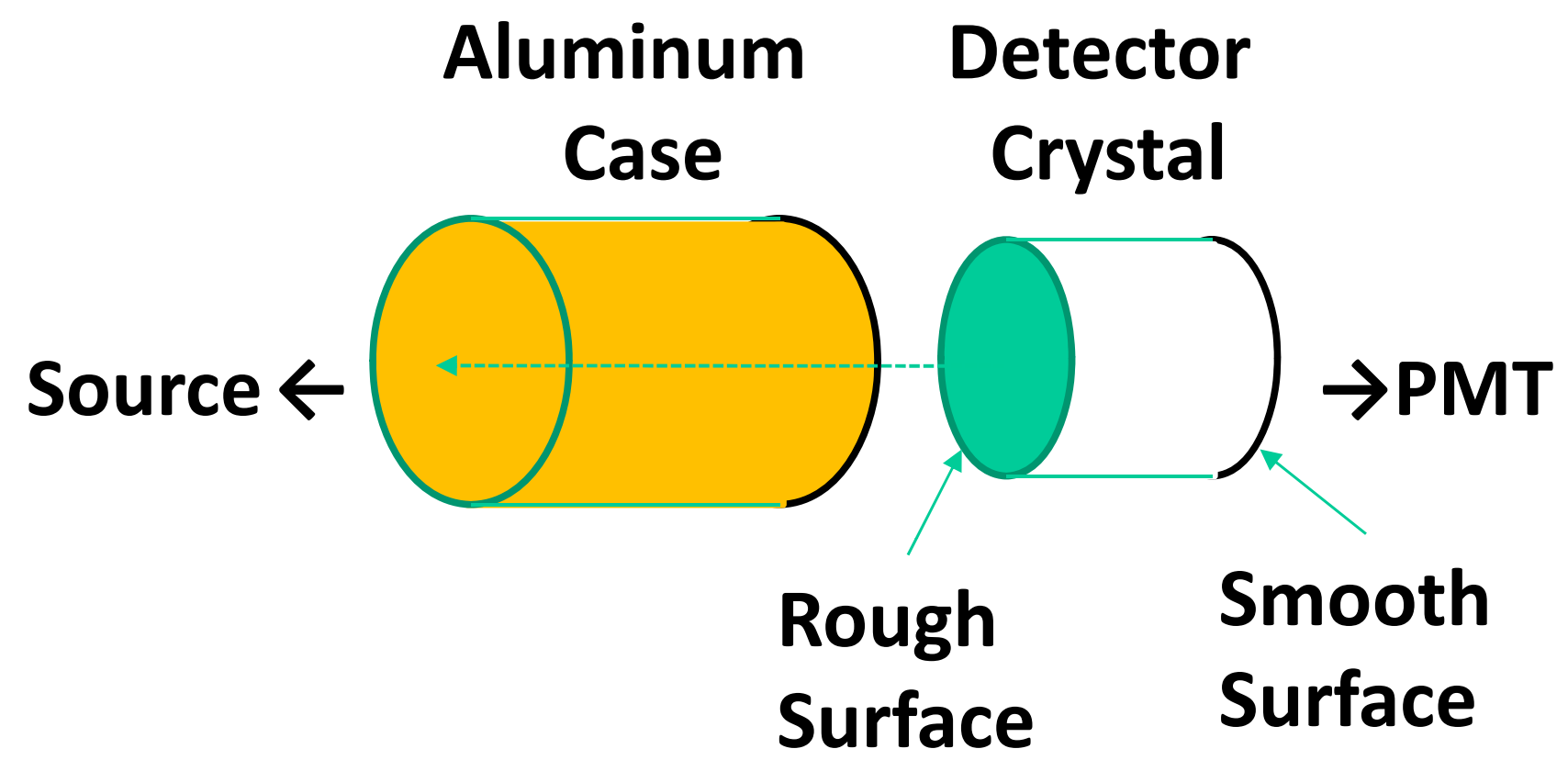




\section{MCNPX Settings \\ Source - Detector Relationship}

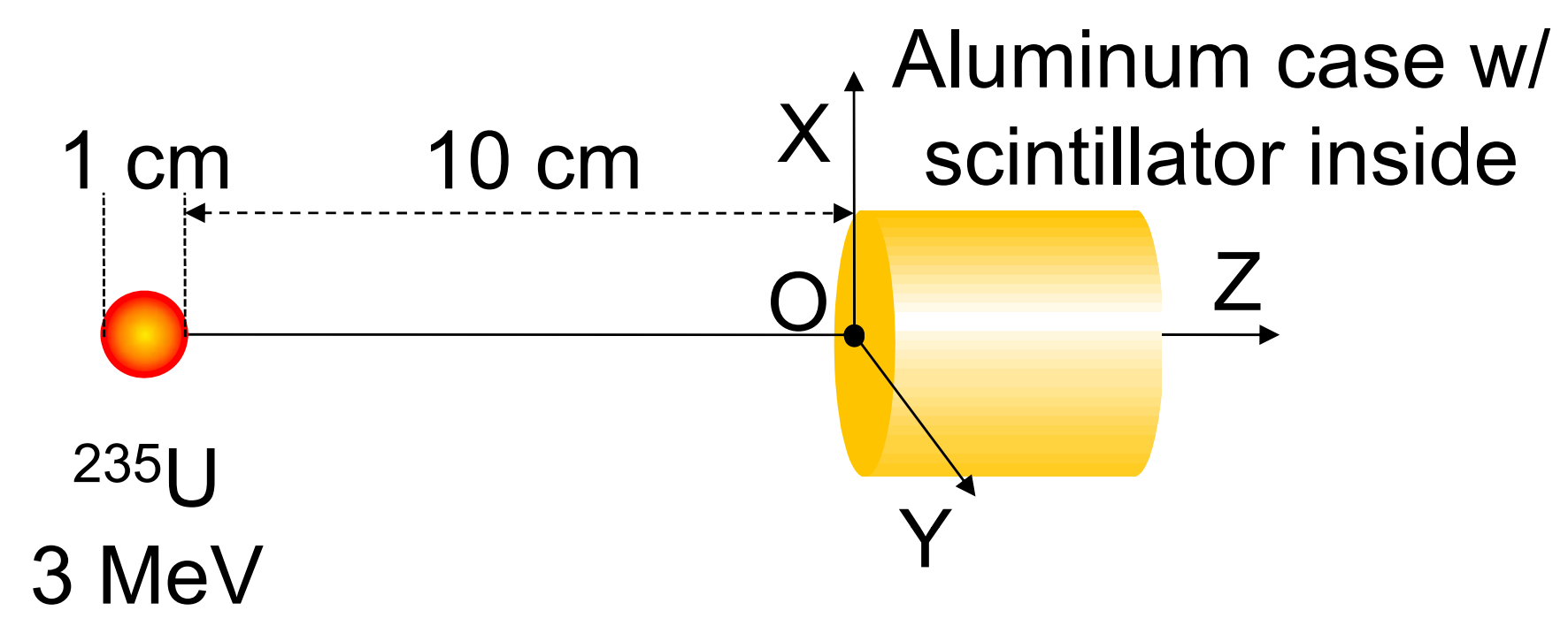




\section{MCNPX Settings Importance Space}

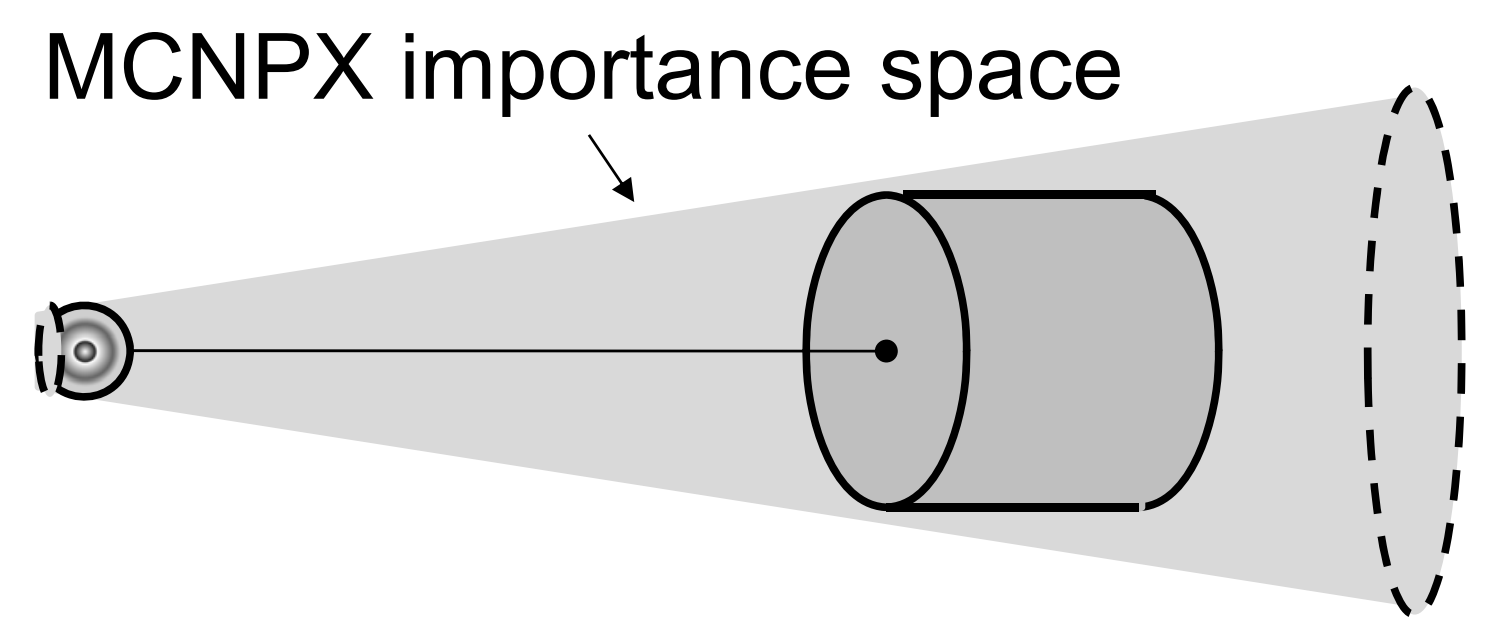




\section{Crystal Surface Geometry Outside of the Box Considerations}

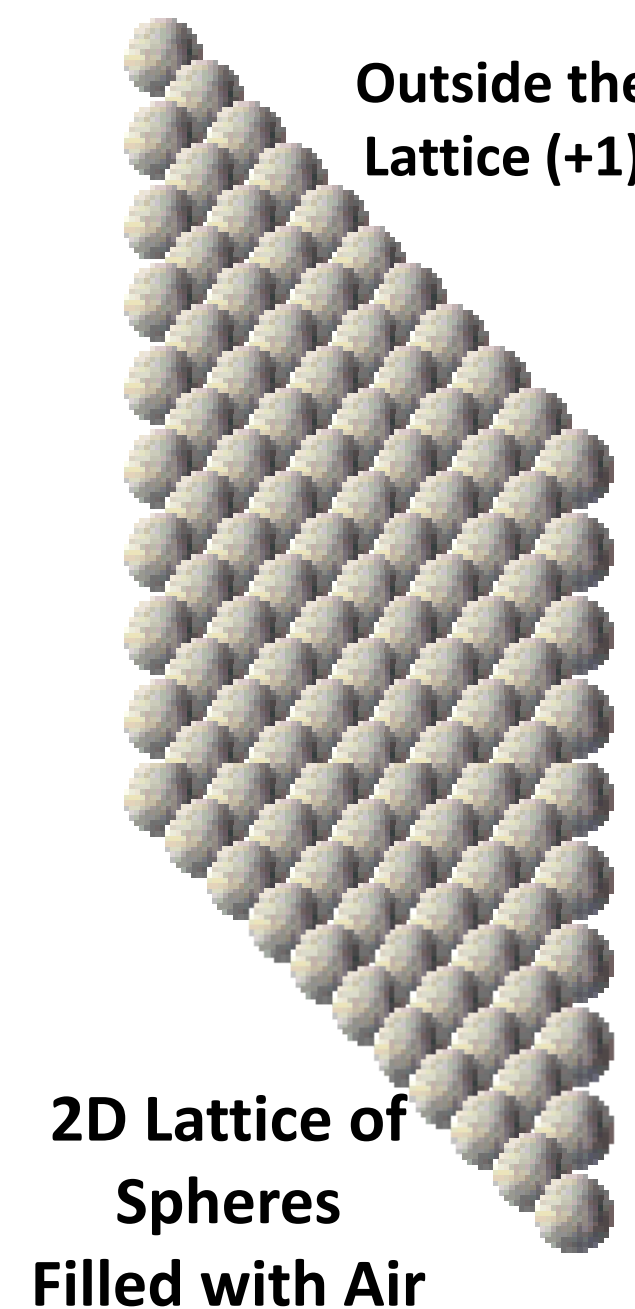
Filled with Air

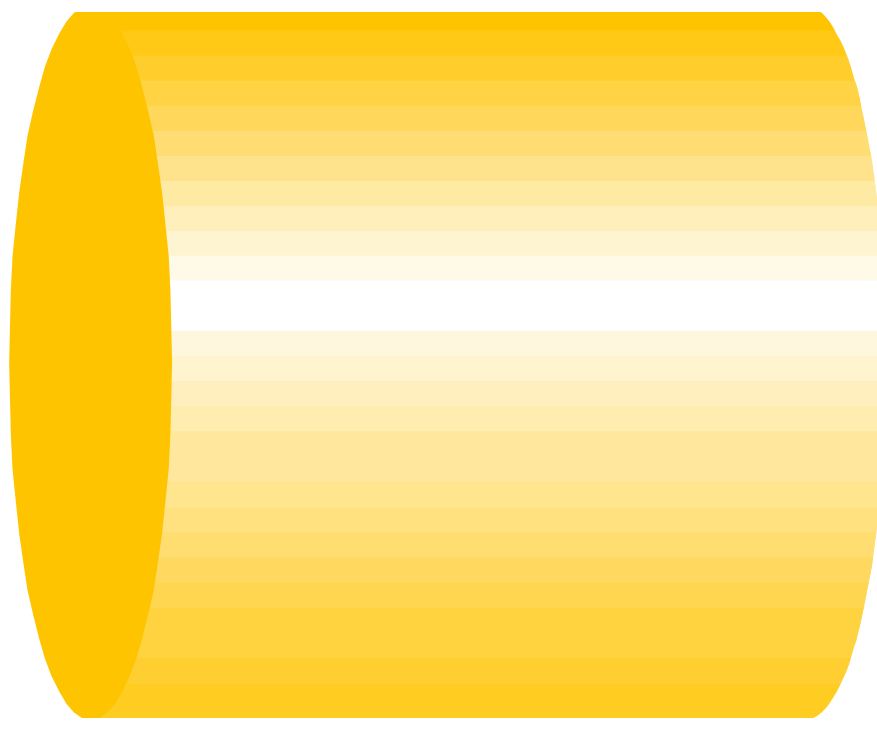

$$
\text { Ф1" X1" }
$$

Cylinder with

Smooth

Surface
A Cylinder with one Rough End

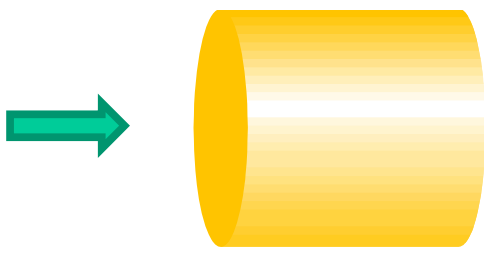

\section{Illustration for} sphere lattice only. Other lattice structures can be handled similarly.

Vision - Service - Partnership 


\section{Consider Three Possible Surface Processing Methods}

- Sanding or Micro-sanding

- Drilling or Micro-drilling

- Cutting or Micro-cutting

- Parallel Cutting

- Cross Cutting

- These processes can be easily adapted by industry, if proven useful

- Correspondent MCNPX modeling schemes will be created 


\section{Crystal Surface Geometry Micro-sanding Pattern}

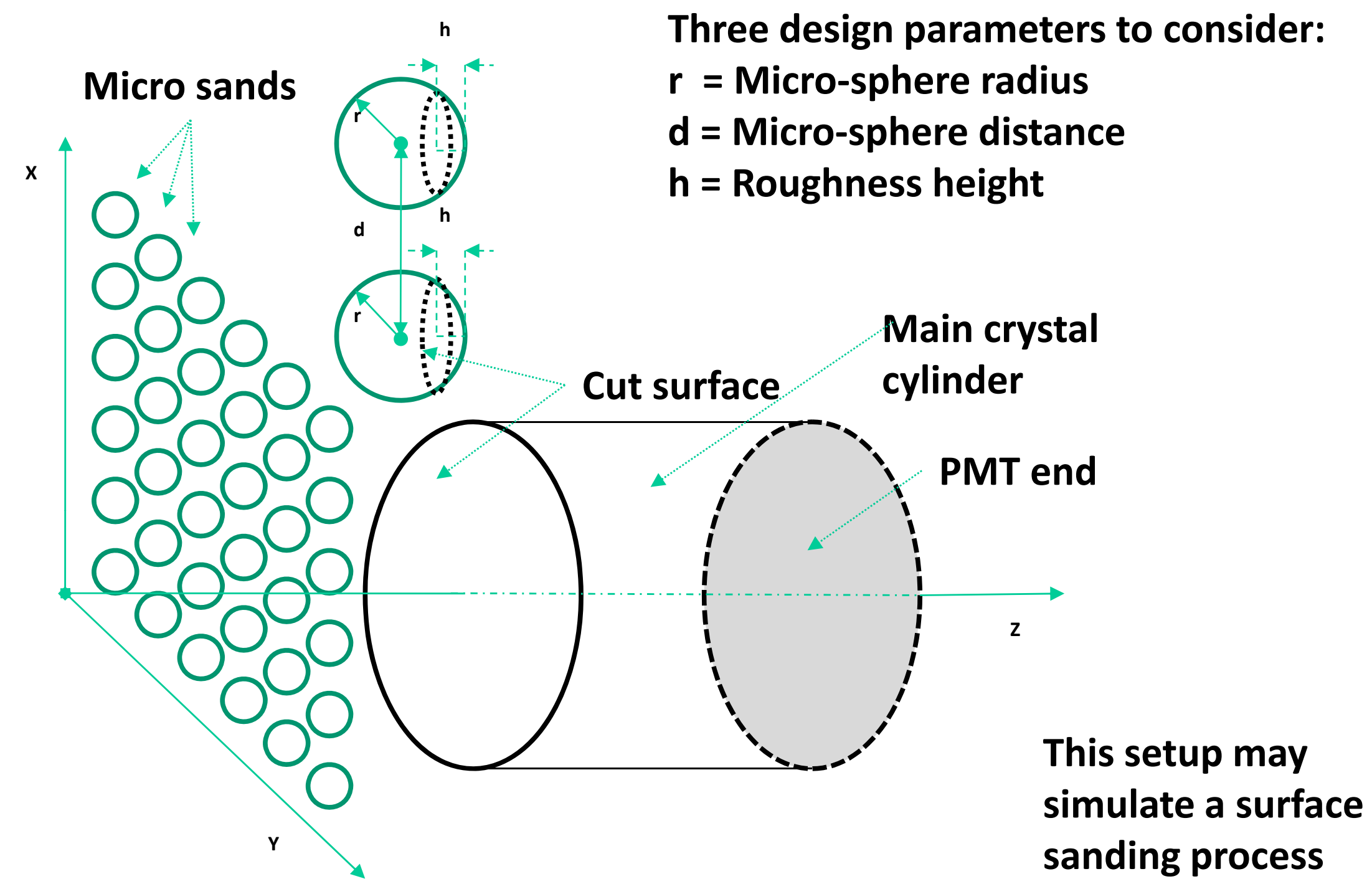




\section{Micro-sanding MCNP Geometrical Parameters}

- For $\mathrm{LaBr}_{3}$ and Nal detectors

- Run parameters with

- Micro-sphere radius $r=100 \mu \mathrm{m}$ and $50 \mu \mathrm{m}$

- Micro-sphere distance $d=4 \times$ sphere radius

- Roughness height $h=1 \times$ sphere radius 


\section{Micro-sanding Modeling Experimental Design Table}

\section{Experimental Parameters and Values}

\begin{tabular}{|c|c|c|c|c|}
\hline $\begin{array}{c}\text { Pattern } \\
\text { Code }\end{array}$ & $\begin{array}{c}\mu \text {-sphere } \\
\text { radius }\end{array}$ & $\begin{array}{c}\mu \text {-sphere } \\
\text { Distance }\end{array}$ & $\begin{array}{c}\mu- \\
\text { Roughness } \\
\text { Height }\end{array}$ & $\begin{array}{c}\# \\
\text { Particles }\end{array}$ \\
\hline Sand050 & $50 \mu \mathrm{m}$ & $200 \mu \mathrm{m}$ & $50 \mu \mathrm{m}$ & $1 \times 10^{6}$ \\
\hline Sand100 & $100 \mu \mathrm{m}$ & $400 \mu \mathrm{m}$ & $100 \mu \mathrm{m}$ & $1 \times 10^{6}$ \\
\hline
\end{tabular}




\section{Crystal Surface Geometry Micro-drilling Pattern}

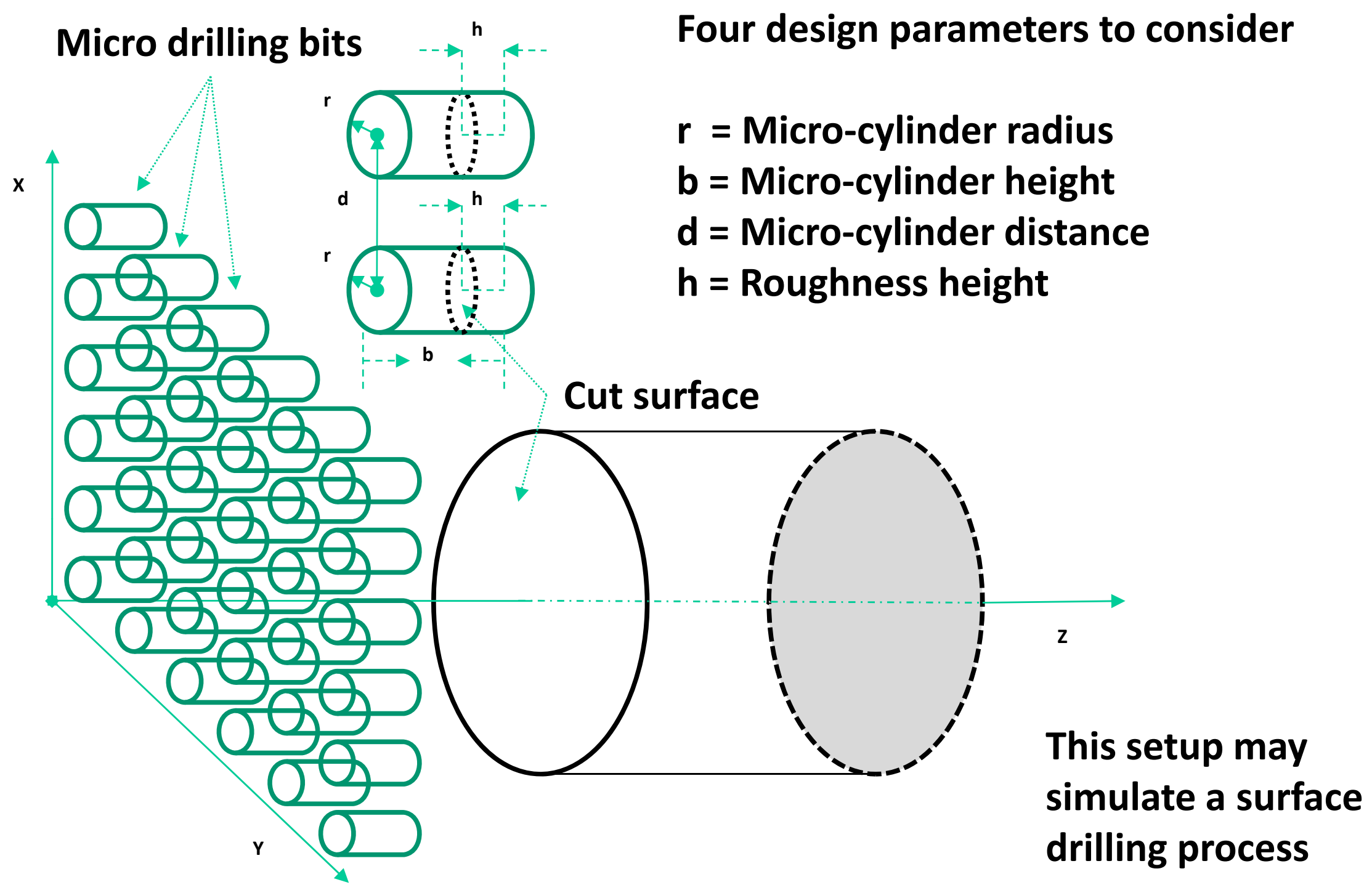




\section{Micro-drilling MCNP Geometrical Parameters}

- For $\mathrm{LaBr}_{3}$ and $\mathrm{Nal}$ detectors

- Run parameters with:

- Micro-cylinder radius $r=100 \mu \mathrm{m}$ and $50 \mu \mathrm{m}$

- Micro-cylinder height $\mathrm{h}=8 \mathrm{x}$ radius

- Micro-cylinder distance $d=4 \times$ sphere radius

- Roughness height $h=4 \times$ sphere radius 


\section{Micro-drilling Modeling Experimental Design Table}

\section{Experimental Parameters and Values}

\begin{tabular}{|c|c|c|c|c|c|}
$\begin{array}{c}\text { Pattern } \\
\text { Code }\end{array}$ & $\begin{array}{c}\mu \text {-cylinder } \\
\text { radius }\end{array}$ & $\begin{array}{c}\mu \text {-cylinder } \\
\text { Distance }\end{array}$ & $\begin{array}{c}\mu \text {-cylinder } \\
\text { Height }\end{array}$ & $\begin{array}{c}\mu \text {-roughness } \\
\text { Height }\end{array}$ & $\begin{array}{c}\# \\
\text { Particles }\end{array}$ \\
\hline Drill050 & $50 \mu \mathrm{m}$ & $200 \mu \mathrm{m}$ & $400 \mu \mathrm{m}$ & $200 \mu \mathrm{m}$ & $1 \times 10^{6}$ \\
\hline Drill100 & $100 \mu \mathrm{m}$ & $400 \mu \mathrm{m}$ & $800 \mu \mathrm{m}$ & $400 \mu \mathrm{m}$ & $1 \times 10^{6}$
\end{tabular}




\section{Crystal Surface Geometry Micro-cutting (Parallel Cutting) Pattern}

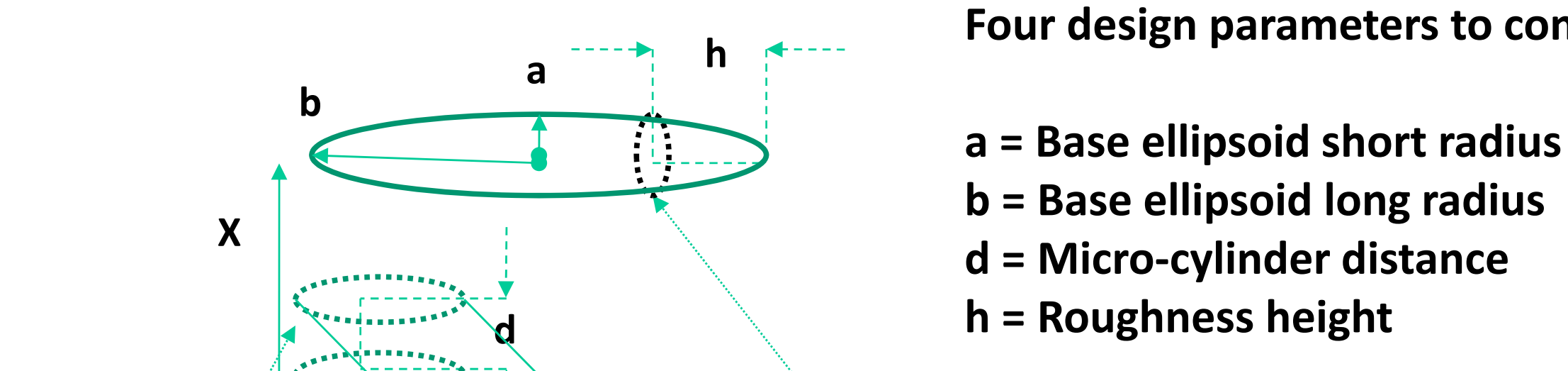

Micro cutting blades
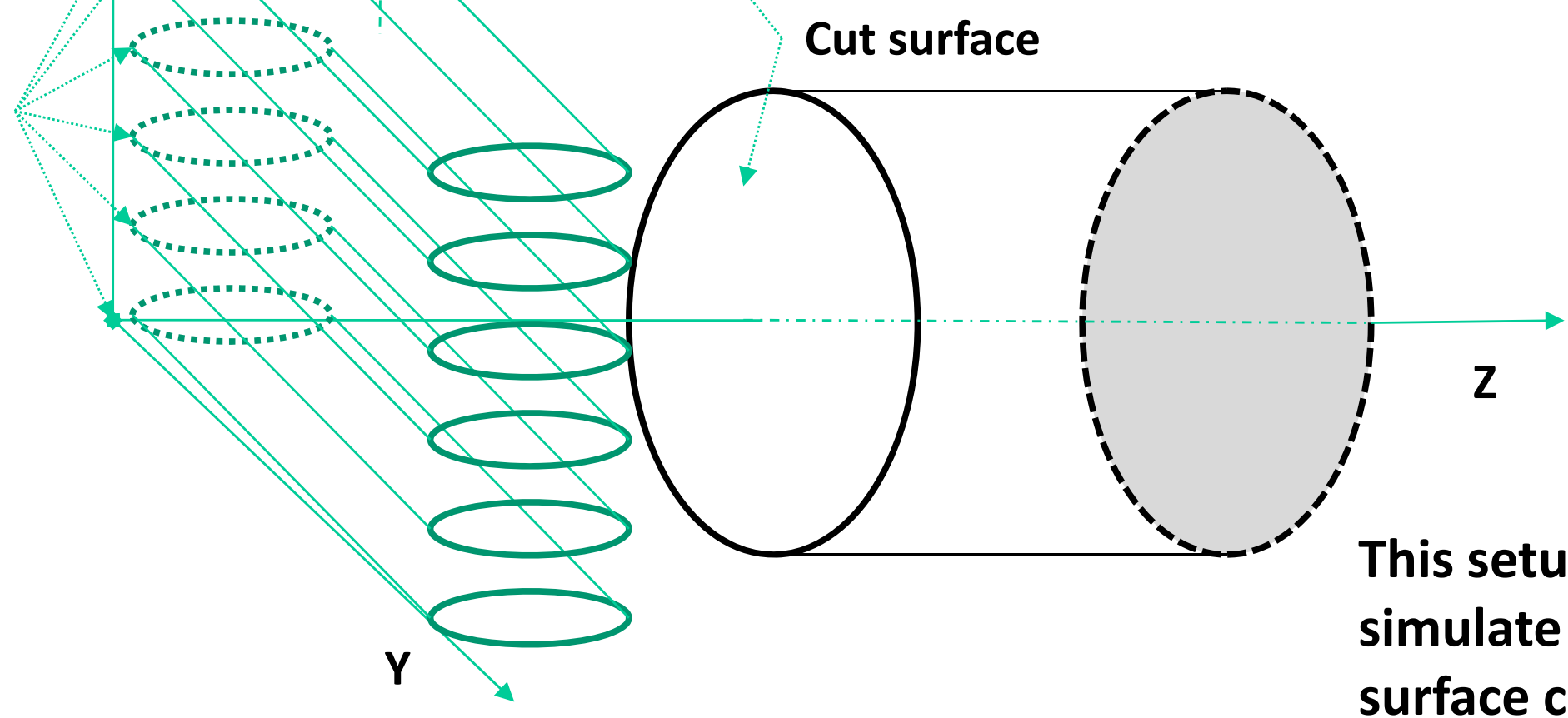

This setup may simulate a parallel surface cutting process 


\section{Micro-cutting (Parallel Cutting) MCNP Geometrical Parameters}

- For $\mathrm{LaBr}_{3}$ and Nal detectors

- Run parameters for the cutting blade with:

- Micro-ellipsoid short radius $a=50 \mu \mathrm{m}$ and long radius $b=100$ $\mu \mathrm{m}$

- Micro-ellipsoid blade length $h=1 "$ (enough for the detector size)

- Micro-ellipsoid blade distance $d=4 \times$ short radius

- Roughness height $h=1 \times$ long radius 


\section{Micro Parallel Cutting Modeling Experimental Design Table}

\section{Experimental Parameters and Values}

\begin{tabular}{|c|c|c|c|c|c|}
\hline $\begin{array}{c}\text { Pattern } \\
\text { Code }\end{array}$ & $\begin{array}{c}\mu \text {-blade } \\
\text { Short radius }\end{array}$ & $\begin{array}{c}\mu \text {-blade } \\
\text { Long radius }\end{array}$ & $\begin{array}{c}\mu \text {-blade } \\
\text { Distance }\end{array}$ & $\begin{array}{c}\mu \text {-rough } \\
\text { Height }\end{array}$ & $\begin{array}{c}\# \\
\text { Particles }\end{array}$ \\
\hline PCut050 & $50 \mu \mathrm{m}$ & $100 \mu \mathrm{m}$ & $200 \mu \mathrm{m}$ & $100 \mu \mathrm{m}$ & $1 \times 10^{6}$ \\
\hline PCut100 & $50 \mu \mathrm{m}$ & $150 \mu \mathrm{m}$ & $200 \mu \mathrm{m}$ & $150 \mu \mathrm{m}$ & $1 \times 10^{6}$ \\
\hline
\end{tabular}




\section{Crystal Surface Geometry Micro-cutting (Cross-Cutting) Pattern}
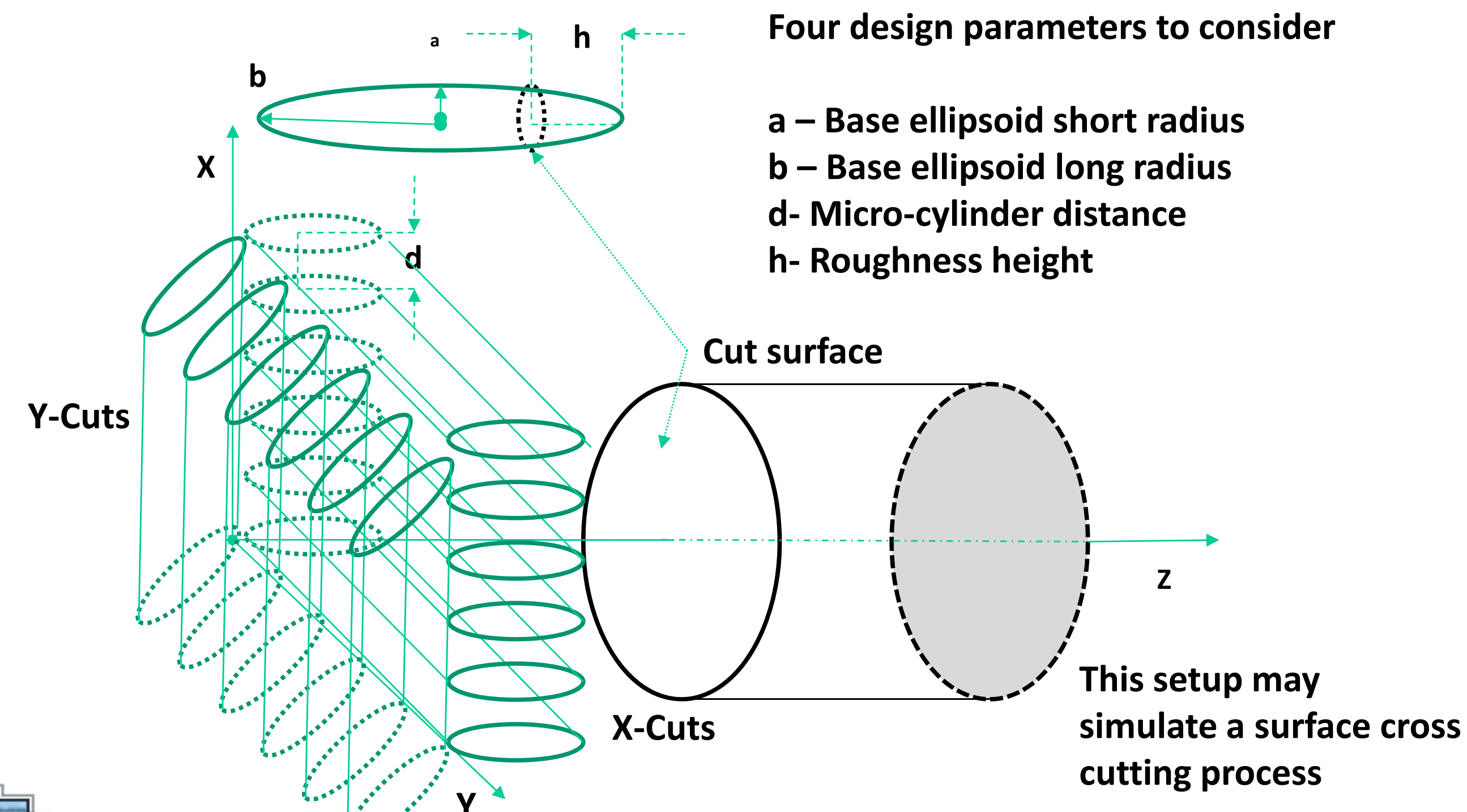

Vision - Service - Partnership 


\section{Micro-Crosscutting Parameters (Cross Cutting - X-cuts followed by Y-cuts)}

- For LaBr3 and Nal detectors

- Run parameters for the cutting blade with:

- Micro-ellipsoid short radius $a=50 \mu \mathrm{m}$ and long radius $b=100$ $\mu \mathrm{m}$

- Micro-ellipsoid blade length $h=1 "$ (enough for the detector size)

- Micro-ellipsoid blade distance $d=4 \times$ short radius

- Roughness height $h=1 \times$ long radius 


\section{Micro Cross Cutting Modeling Experimental Design Table}

\begin{tabular}{|c|c|c|c|c|c|}
\hline \multicolumn{6}{|c|}{ Experimental Parameters and Values } \\
\hline $\begin{array}{l}\text { Pattern } \\
\text { Code }\end{array}$ & $\begin{array}{c}\mu \text {-blade } \\
\text { Short radius }\end{array}$ & $\begin{array}{c}\mu \text {-blade } \\
\text { Long radius }\end{array}$ & $\begin{array}{l}\mu \text {-blade } \\
\text { Distance }\end{array}$ & $\begin{array}{l}\mu \text {-rough } \\
\text { Height }\end{array}$ & $\begin{array}{c}\# \\
\text { Particles }\end{array}$ \\
\hline Cut050 & $50 \mu \mathrm{m}$ & $100 \mu \mathrm{m}$ & $200 \mu \mathrm{m}$ & $100 \mu \mathrm{m}$ & $1 \times 10^{6}$ \\
\hline Cut1c & $50 \mu \mathrm{m}$ & $150 \mu \mathrm{m}$ & $200 \mu \mathrm{m}$ & $150 \mu \mathrm{m}$ & $1 \times 10^{6}$ \\
\hline
\end{tabular}




\section{Energy Deposition Summary}

F6 Tally (MCNPX)

\begin{tabular}{|c|c|c|c|c|c|c|c|c|}
\hline $\begin{array}{c}\text { Surface } \\
\text { type }\end{array}$ & CdSe & $\mathrm{CeBr}_{3}$ & $\mathrm{CeF}_{3}$ & $\mathrm{LaBr}_{3}$ & $\mathrm{LaCl}_{3}$ & $\mathrm{LaF}_{3}$ & Nal & $\mathrm{SiO}_{2}$ \\
\hline Smooth & $\begin{array}{r}.6941 \mathrm{E}- \\
05 \\
\end{array}$ & $\begin{array}{r}2.7244 \mathrm{E}- \\
05 \\
\end{array}$ & $\begin{array}{r}2.7610 \mathrm{E}- \\
05 \\
\end{array}$ & $\begin{array}{r}2.7097 \mathrm{E}- \\
05 \\
\end{array}$ & $\begin{array}{r}2.7752 \mathrm{E}- \\
05 \\
\end{array}$ & $\begin{array}{r}2.7645 \mathrm{E}- \\
05 \\
\end{array}$ & $\begin{array}{r}2.8022 \mathrm{E}- \\
05 \\
\end{array}$ & $\begin{array}{r}2.6710 \mathrm{E} \\
05 \\
\end{array}$ \\
\hline Sand050 & $\begin{array}{r}2.6927 \mathrm{E}- \\
05\end{array}$ & $\begin{array}{r}2.7458 \mathrm{E}- \\
05\end{array}$ & $\begin{array}{r}2.8072 \mathrm{E}- \\
05\end{array}$ & $\begin{array}{r}2.7559 \mathrm{E}- \\
05\end{array}$ & $\begin{array}{r}2.8134 \mathrm{E}- \\
05\end{array}$ & $\begin{array}{r}2.7831 \mathrm{E}- \\
05\end{array}$ & $\begin{array}{r}2.9260 \mathrm{E}- \\
05\end{array}$ & $\begin{array}{r}2.7761 \mathrm{E}- \\
05\end{array}$ \\
\hline Sand100 & $\begin{array}{r}2.6630 \mathrm{E}- \\
05\end{array}$ & $\begin{array}{r}2.7125 \mathrm{E}- \\
05 \\
\end{array}$ & $\begin{array}{r}2.7644 \mathrm{E}- \\
05 \\
\end{array}$ & $\begin{array}{r}2.7227 \mathrm{E}- \\
05 \\
\end{array}$ & $\begin{array}{r}2.7222 \mathrm{E}- \\
05 \\
\end{array}$ & $\begin{array}{r}2.7364 \mathrm{E}- \\
05 \\
\end{array}$ & $\begin{array}{r}2.8346 \mathrm{E}- \\
05 \\
\end{array}$ & $\begin{array}{r}2.6899 \mathrm{E} \\
05 \\
\end{array}$ \\
\hline Drill050 & $\begin{array}{r}2.7299 \mathrm{E}- \\
05 \\
\end{array}$ & $\begin{array}{r}2.7810 \mathrm{E}- \\
05 \\
\end{array}$ & $\begin{array}{r}2.8585 \mathrm{E}- \\
05 \\
\end{array}$ & $\begin{array}{r}2.7710 \mathrm{E}- \\
05 \\
\end{array}$ & $\begin{array}{r}2.8442 \mathrm{E}- \\
05 \\
\end{array}$ & $\begin{array}{r}2.8077 \mathrm{E}- \\
05 \\
\end{array}$ & $\begin{array}{r}2.9307 \mathrm{E}- \\
05 \\
\end{array}$ & $\begin{array}{r}2.8537 \mathrm{E}- \\
05 \\
\end{array}$ \\
\hline Drill100 & $\begin{array}{r}2.7526 \mathrm{E}- \\
05\end{array}$ & $\begin{array}{r}2.8157 \mathrm{E}- \\
05\end{array}$ & $\begin{array}{r}2.8404 \mathrm{E}- \\
05\end{array}$ & $\begin{array}{r}2.7907 \mathrm{E}- \\
05\end{array}$ & $\begin{array}{r}2.8568 \mathrm{E}- \\
05\end{array}$ & $\begin{array}{r}2.8262 \mathrm{E}- \\
05\end{array}$ & $\begin{array}{r}2.9099 \mathrm{E}- \\
05\end{array}$ & $\begin{array}{r}2.7993 \mathrm{E} \\
05\end{array}$ \\
\hline $\mathrm{PCu}$ & $\begin{array}{r}2.7804 \mathrm{E}- \\
05 \\
\end{array}$ & $\begin{array}{r}2.7862 \mathrm{E}- \\
05 \\
\end{array}$ & $\begin{array}{r}2.8874 \mathrm{E}- \\
05 \\
\end{array}$ & $\begin{array}{r}2.7960 \mathrm{E}- \\
05 \\
\end{array}$ & $\begin{array}{r}2.8256 \mathrm{E}- \\
05 \\
\end{array}$ & $\begin{array}{r}2.8682 \mathrm{E}- \\
05 \\
\end{array}$ & $\begin{array}{r}2.9688 \mathrm{E}- \\
05 \\
\end{array}$ & $\begin{array}{r}2.8133 \mathrm{E} \\
05 \\
\end{array}$ \\
\hline 00 & $\begin{array}{r}2.8637 \mathrm{E}- \\
05\end{array}$ & $\begin{array}{r}2.8808 \mathrm{E}- \\
05\end{array}$ & $\begin{array}{r}2.9482 \mathrm{E}- \\
05\end{array}$ & $\begin{array}{r}2.8714 \mathrm{E}- \\
05\end{array}$ & $\begin{array}{r}2.8730 \mathrm{E}- \\
05\end{array}$ & $\begin{array}{r}2.9171 \mathrm{E}- \\
05\end{array}$ & $\begin{array}{r}2.9218 \mathrm{E}- \\
05\end{array}$ & $\begin{array}{r}2.9048 \mathrm{E} \\
05\end{array}$ \\
\hline XCut050 & $\begin{array}{r}2.6934 \mathrm{E}- \\
05\end{array}$ & $\begin{array}{r}2.7491 \mathrm{E}- \\
05 \\
\end{array}$ & $\begin{array}{r}2.7878 \mathrm{E}- \\
05 \\
\end{array}$ & $\begin{array}{r}2.7281 \mathrm{E}- \\
05 \\
\end{array}$ & $\begin{array}{r}2.7510 \mathrm{E}- \\
05 \\
\end{array}$ & $\begin{array}{r}2.7874 \mathrm{E}- \\
05 \\
\end{array}$ & $\begin{array}{r}2.9070 \mathrm{E}- \\
05 \\
\end{array}$ & $\begin{array}{r}2.7650 \mathrm{E} \\
05 \\
\end{array}$ \\
\hline XCut100 & $\begin{array}{r}2.7147 \mathrm{E}- \\
05\end{array}$ & $\begin{array}{r}2.7836 \mathrm{E}- \\
05\end{array}$ & $\begin{array}{r}2.7984 \mathrm{E}- \\
05\end{array}$ & $\begin{array}{r}2.7527 \mathrm{E}- \\
05\end{array}$ & $\begin{array}{r}2.7970 \mathrm{E}- \\
05\end{array}$ & $\begin{array}{r}2.7657 \mathrm{E}- \\
05\end{array}$ & $\begin{array}{r}2.8786 \mathrm{E}- \\
05\end{array}$ & $\begin{array}{r}2.7433 \mathrm{E} \\
05\end{array}$ \\
\hline
\end{tabular}




\section{Average Energy Deposition}

- 3D view of the data in the previous slide

- Front line shows the data for smooth surfaces

\section{Average Energy Deposition (Mev/g)}

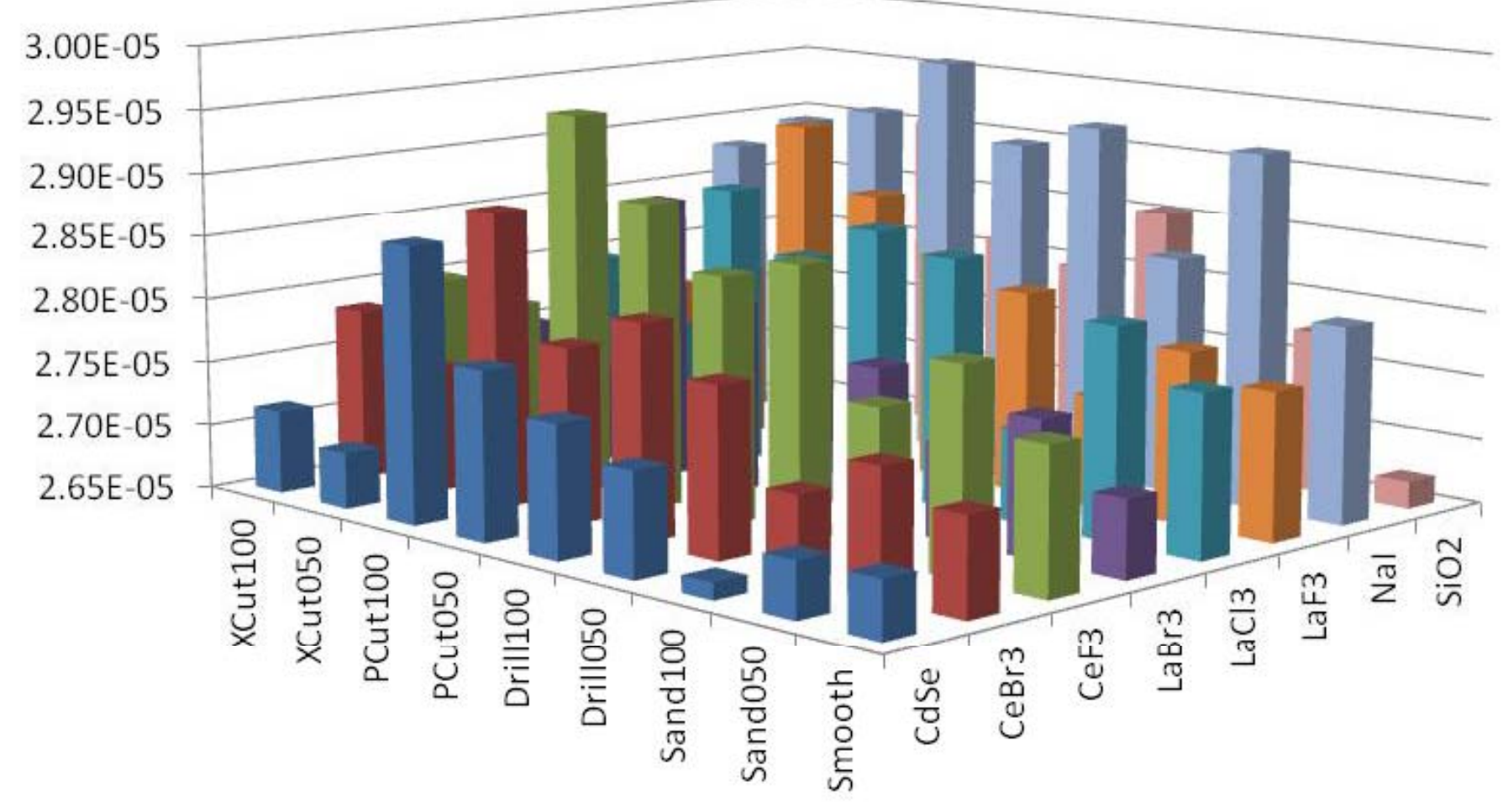




\section{Difference in Energy Deposition}

- Differences in average energy deposition as compared with correspondent crystals with smooth surfaces

- Front line data are zeroes for smooth surfaces.

Difference in Average Energy Deposition (Mev/g) Between Rough and Smooth Scintillators

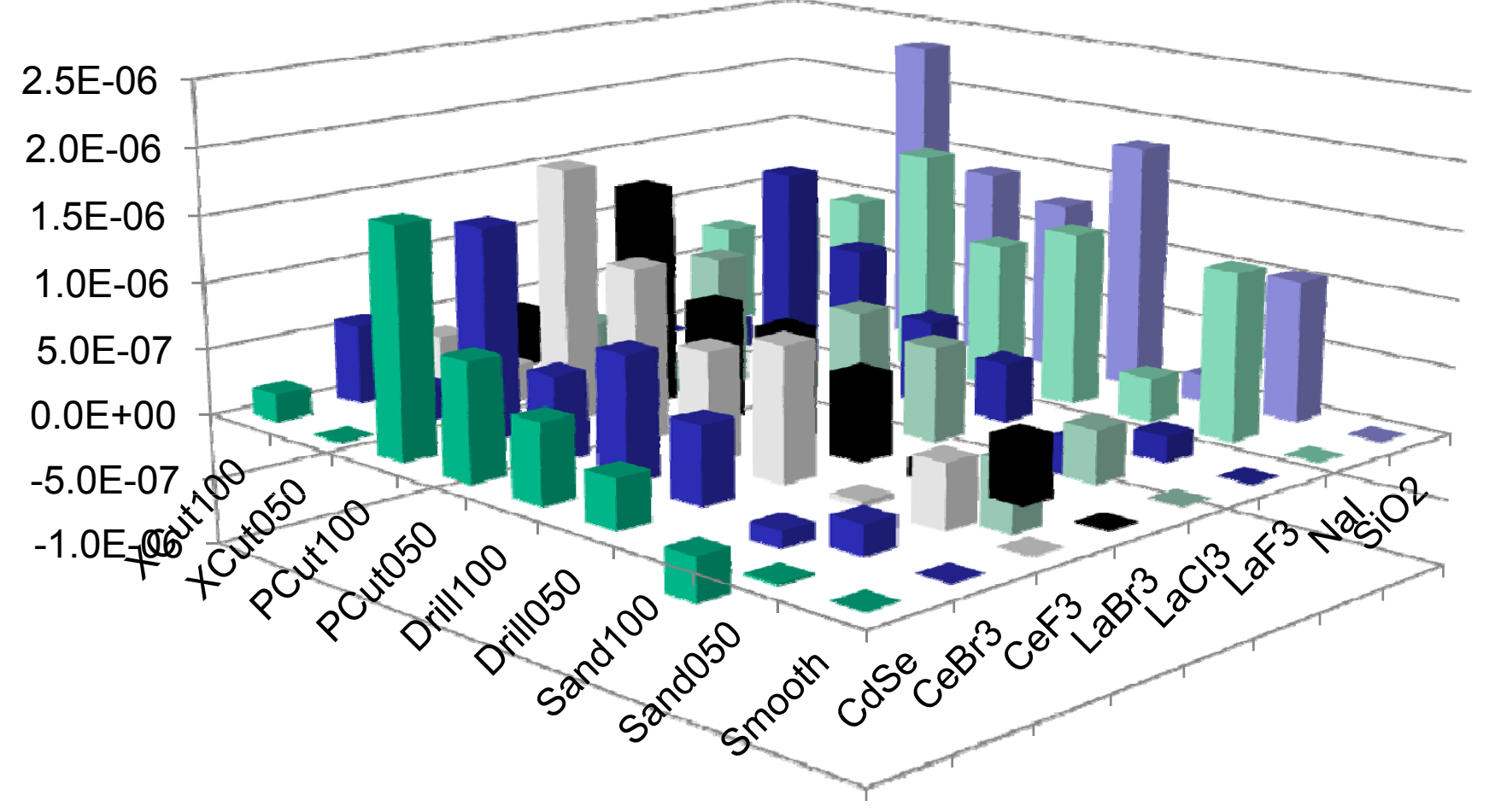




\section{Energy Deposition Improvement}

\section{Energy deposition improvement as compared with crystals with smooth surfaces}

\begin{tabular}{|lcccccccc|cccc|}
\hline Improve\% & CdSe & CeBr3 & CeF3 & LaBr3 & LaCl3 & LaF3 & Nal & SiO2 & Rank & Min & Mean & Max \\
Smooth & $0.00 \%$ & $0.00 \%$ & $0.00 \%$ & $0.00 \%$ & $0.00 \%$ & $0.00 \%$ & $0.00 \%$ & $0.00 \%$ & 8 & $0.00 \%$ & $0.00 \%$ & $0.00 \%$ \\
Sand050 & $-0.05 \%$ & $0.78 \%$ & $1.67 \%$ & $1.71 \%$ & $1.38 \%$ & $0.67 \%$ & $4.42 \%$ & $3.94 \%$ & 5 & $-0.05 \%$ & $1.81 \%$ & $4.42 \%$ \\
Sand100 & $-1.16 \%$ & $-0.44 \%$ & $0.13 \%$ & $0.48 \%$ & $-1.91 \%$ & $-1.02 \%$ & $1.15 \%$ & $0.71 \%$ & $9: *$ & $-1.91 \%$ & $-0.26 \%$ & $1.15 \%$ \\
Drill050 & $1.33 \%$ & $2.08 \%$ & $3.53 \%$ & $2.26 \%$ & $2.49 \%$ & $1.56 \%$ & $4.58 \%$ & $6.84 \%$ & 4 & $1.33 \%$ & $3.08 \%$ & $6.84 \%$ \\
Drill100 & $2.17 \%$ & $3.35 \%$ & $2.88 \%$ & $2.99 \%$ & $2.94 \%$ & $2.23 \%$ & $3.84 \%$ & $4.80 \%$ & 3 & $2.17 \%$ & $3.15 \%$ & $4.80 \%$ \\
PCut050 & $3.20 \%$ & $2.27 \%$ & $4.58 \%$ & $3.19 \%$ & $1.82 \%$ & $3.75 \%$ & $5.94 \%$ & $5.33 \%$ & 2 & $1.82 \%$ & $3.76 \%$ & $5.94 \%$ \\
PCut100 & $6.30 \%$ & $5.74 \%$ & $6.78 \%$ & $5.97 \%$ & $3.53 \%$ & $5.52 \%$ & $4.27 \%$ & $8.75 \%$ & $1 \%$ & $3.53 \%$ & $5.86 \%$ & $8.75 \%$ \\
XCut050 & $-0.02 \%$ & $0.91 \%$ & $0.97 \%$ & $0.68 \%$ & $-0.87 \%$ & $0.83 \%$ & $3.74 \%$ & $3.52 \%$ & 7 & $-0.87 \%$ & $1.22 \%$ & $3.74 \%$ \\
XCut100 & $0.77 \%$ & $2.17 \%$ & $1.36 \%$ & $1.59 \%$ & $0.79 \%$ & $0.04 \%$ & $2.73 \%$ & $2.71 \%$ & 6 & $0.04 \%$ & $1.52 \%$ & $2.73 \%$ \\
\hline Rank & 7 & 5 & 3 & 4 & $8 \%$ & 6 & 2 & $1 . ;$ & Global & $-1.91 \%$ & $2.24 \%$ & $8.75 \%$ \\
Min & $-1.16 \%$ & $-0.44 \%$ & $0.00 \%$ & $0.00 \%$ & $-1.91 \%$ & $-1.02 \%$ & $0.00 \%$ & $0.00 \%$ & $-1.91 \%$ & & Yellow & $=0$ \\
Mean & $1.39 \%$ & $1.87 \%$ & $2.43 \%$ & $2.10 \%$ & $1.13 \%$ & $1.51 \%$ & $3.41 \%$ & $4.07 \%$ & $2.24 \%$ & & Green & $>0$ \\
Max & $6.30 \%$ & $5.74 \%$ & $6.78 \%$ & $5.97 \%$ & $3.53 \%$ & $5.52 \%$ & $5.94 \%$ & $8.75 \%$ & $8.75 \%$ & & Red & $<0$ \\
\hline
\end{tabular}




\section{Percentage Improvement in Energy Deposition}

- 3D view of data in previous slide

- Shows percentage improvement as compared with correspondent smooth surfaces

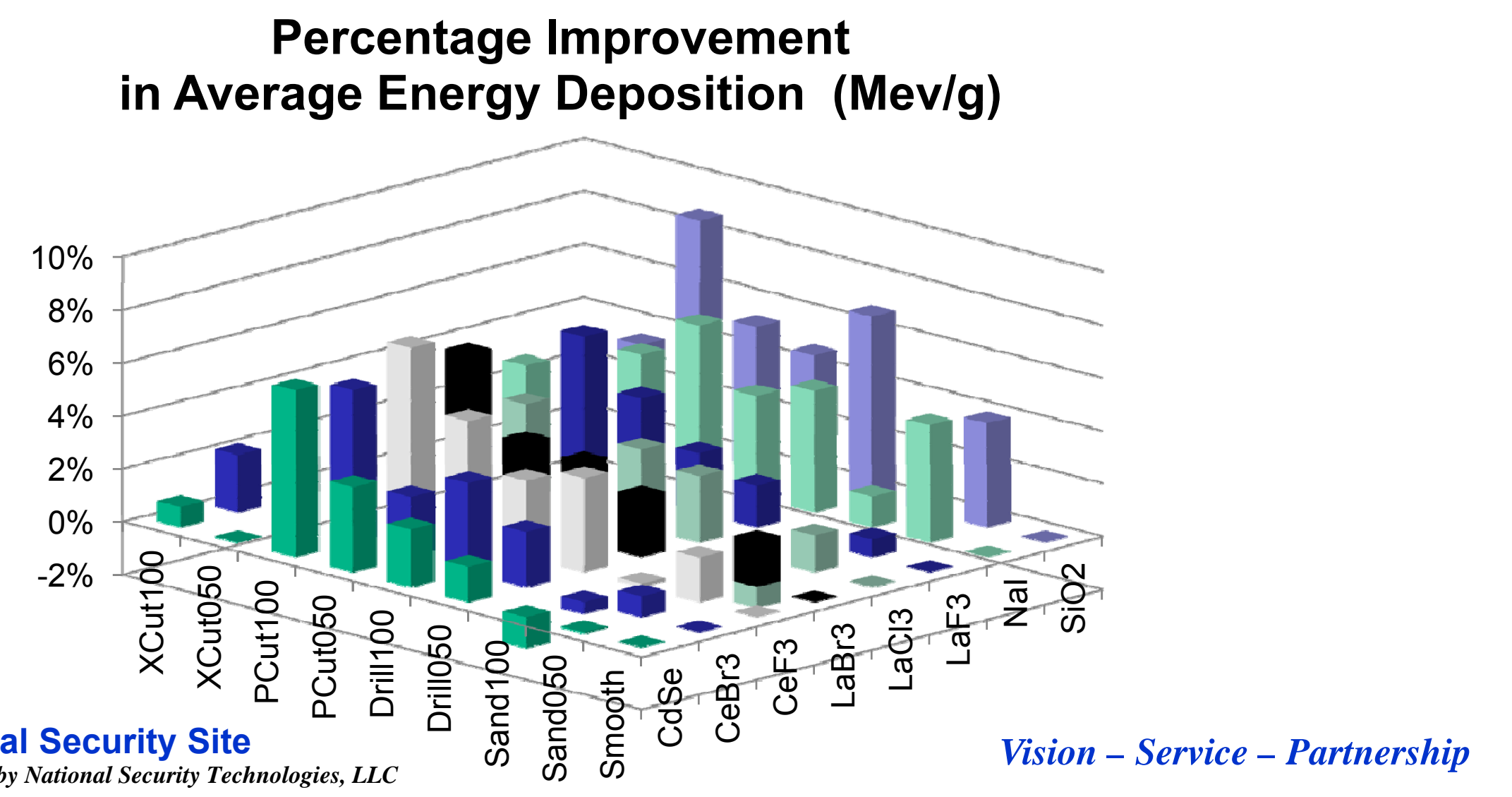




\section{Discussion}

- MCNPX results suggest that a rough surface generally improves the detectors photon detection efficiency

- Of all 64 rough surfaces and scintillator couplings, about 57 showed some improvement (89\%)

- The rough surfaces demonstrated systematic advantages for scintillators $\mathrm{Nal}, \mathrm{LaBr}_{3}, \mathrm{LaCl}_{3}, \mathrm{CeBr}_{3}$, and $\mathrm{LaF}_{3}$

- The mean improvement is about $2.5 \%$ for all crystals, with a maximum improvement of $8.75 \%$ for $\mathrm{SiO}_{2}$ 


\section{Discussion}

Reasons for the these types of simulation results are not explained by this work; however, the following are suspected:

- Increased gamma capturing may be due to the periodicities (or wave patterns) of the evaluated roughness patterns, as compared with those studied in optics for diffraction gratings or other fields.

- Increased gamma capturing may be to due to the increase of the effective surface areas of the scintillators under various surface-roughness schemes.

- The increase in internal reflection of the photons in the visible wavelengths resulting from scintillation may be an outcome or benefit of roughening the crystal surface. 


\section{Discussion}

\section{Caveats:}

- Statistical uncertainties in real-world data and in these MCNPX simulations are present. A high precision statistical analysis is required to ascertain the true statistical significance of these findings.

- The MCNPX results presented here are for tallies of energy deposition.

- The effects of rough surfaces on photon internal reflection have not yet been studied.

- A more detailed analysis that incorporates the incoming gamma-ray energy deposition, internal reflection of visible photons, depth of interaction, and rough surfaces on photon internal reflection, etc., is required in order to explain the overall comprehensive picture. 


\section{Summary}

- We have reported on our recent MCNPX simulation results of energy deposition for a group of 8 scintillation detectors, coupled with various rough surface patterns.

- The MCNPX results generally favored the detectors with various rough surface patterns.

- The observed MCNPX results are not fully explained by this work.

- The MCNPX results are reported for stimulating further investigations. 